\title{
OWL site survey: First seeing measurement with ADIMM
}

\author{
Benkhaldoun Z. Author $1^{a}$, Abahamid A. Author2 ${ }^{a}$, \\ El Azhari Y. Author $3^{a}$ and Siher E. Author $4^{a}$ \\ ${ }^{a}$ Laboratoire de Physique des Hautes Energies et Astrophysique, Département de Physique, \\ Faculté des Sciences Semlalia, BP: 2390, Marrakech, Maroc
}

\begin{abstract}
The ESO OWL site survey plan includes the analysis of the astronomical quality of the Atlas mountains in Morocco. In this paper we are preseningt the first long time measurement of optical turbulence at Oukaimeden site. For this work we built an instrument called ADIMM for an Automated Differencial Image Motion Monitor, and we use it to the measure the optical turbulence at the Oukaimeden Site. We are describing the instrument and reporting the first results obtained after six month of working on this project. The results of night-time seeing measurements carried out during the period from Jun 14 up to October 012003 are presented. The median and mean values of the seeing for the entire period of observations are respectively 0.75 " and 0.84 ".

This work was organized in the framework of contract Num.69651/ODG/02/9005/GWI between the European Southern Observatory (ESO) and Astrophysics and Physics of High Energy Laboratory of the Faculty of Sciences, Cadi Ayyad University (LPHEA).
\end{abstract}

Keywords: Optical Turbulence, Seeing, ADIMM, Site Testing.

\section{INTRODUCTION}

The Oukaïmeden site is located in the Moroccan high Atlas $\left(7^{\circ} 52^{\prime} 52^{\prime \prime} \mathrm{W}, 31^{\circ} 12^{\prime} 32^{\prime \prime} \mathrm{N}, 2700 \mathrm{~m}\right), 70 \mathrm{~km}$ far from Marrakesh city. Several site testing campaign has been performed at Oukaïmeden. The results of this study support the following conclusions (Benkhaldoun 2002): The mean night-time temperature is moderate all year long. Extreme temperature rarely seem to occur and the stability of the night-time temperature is consistent. The wind should not constitute a major problem for astronomical observations since the average wind speed is normally low. The predominant wind direction is from the SE during the night and from WNW during the day. This is not harmful, given the position of the site with respect to the high Atlas Mountains in SE and SW directions. Local humidity is almost always modest. The atmospheric extinction is comparable to the best sites in the world (Benkhaldoun et al. 1993, 1998, 2002; Siher et al. 2003).

On the other hand, the main atmospheric optical parameters (AOP) has been measured during 10 nights in April 1998 with the GSM instrument. Ziad 2001 published the main results of this campaign. The seeing data measured by the GSM, presents some excellent nights but during some other nights the seeing was worse. For this parameter further measurements are needed for a long period to provide more statistics and to know the variability versus seasons.

We are developing a new seeing monitor in the Astrophysics and Physics of High Energy Laboratory of Cadi Ayyad University. It is installed on the Oukaïmeden site for a long period in order to appraise different parameters of the atmospheric turbulence, such as Scintillation Index, Extinction coefficient and Seeing (FWHM). Accurate, absolute, and reproductible measurements are the expected qualities of any instrument. Our automated differential image motion monitor (ADIMM), is presented which assesses astronomical seeing with those requirements. It has been designed also to be reliable, simple, transportable, low cost, and easy to set up, to operate, and to maintain. Its accuracy has been studied, both from theoretical and experimental points of view, mainly with respect to CCD sampling manufactured by SBIG, photon noise, and threshold influence on centroid determination. It monitors the seeing with a temporal resolution better than one minute. this type of instrument is essential for testing new and already existing sites and to ascertain that the surroundings of telescopes do not generate additional turbulence.

Further author information: (Send correspondence to B. Z.)

B. Z.: E-mail: zouhair@ucam.ac.ma, Telephone: 21244434649

A. A.: E-mail: a.abahamid@ucam.ac.ma, Telephone: 21244434649 


\section{DESCRIPTION OF THE ADIMM}

Atmospheric turbulence is the most important factor limiting the resolution of the optical instruments. For telescopes of large diameters, the quality of the observations is very dependent on the conditions of turbulence. Precise and quantified knowledge optical effects of atmospheric turbulence is essential to optimize the observations in High Angular Resolution and to select the best astronomical sites. We are presenting here, a new generation of an Automated Differential Image Motion Monitor (ADIMM) simple, transportable and cheap (thanks to the type of the camera used). Camera CCD (Charge Coupled Device) of type SBIG (Santa Barbara Instrument Group), is adapted for the measurement of the "seeing" which characterizes the astronomical degradation of the images by optical turbulence.

We have developed a software (DIMMAR), which will make it possible to command both an Ultima 2000 Celestron Telescope and the SBIG camera. And moreover it will make it possible to follow the temporal variability of the intensity of optical turbulence along the atmosphere.

The presence of turbulence in the Earth's atmosphere causes the stellar image formed by a telescope of small diameter to move randomly. The position of the stellar image in the focal plane is directly related to the tilt angle of the wavefront with regard to the entrance pupil plane of the telescope. According to theoretical calculations, the atmospheric turbulence can be estimated through the variance of the image motion.

Image quality through a telescope is directly related to the statistics of the perturbations of the incoming wavefront. The DIMM method consists on measuring wavefront slope differences over two small pupils some distance apart. Because it is a differential method, the technique is inherently insensitive to tracking errors and wind shake. In practice, starlight goes through two small circular sub-apertures, cut in mask placed at the entrance of a small telescope. One of the sub-apertures contains a prism in order to create a second image of the star on the detector. The dual star images obtained exhibits a relative motion in the image plane that represents the local wavefront tilts, which can be expressed in terms of an absolute seeing scale according to the approximate formula:

$$
F W H M=0.976 \frac{\lambda}{r_{o}}
$$

where $\lambda$ is the wavelength and $r_{o}$ is the Fried parameter.

For a given wavefront of coherence radius $r_{o}$ the variance of the differential motion (in radian $^{2}$ ) between two pupils of diameter $D$ at the distance $d$ of each other depends on the direction of measurement (Sarazin et al. 1990):

$$
\begin{aligned}
& \sigma_{\|}^{2} \simeq 2\left(0.179 D^{-1 / 3}-0.0968 d^{-1 / 3}\right) \lambda^{2} r_{o}^{-5 / 3} \\
& \sigma_{\perp}^{2} \simeq 2\left(0.179 D^{-1 / 3}-0.145 d^{-1 / 3}\right) \lambda^{2} r_{o}^{-5 / 3}
\end{aligned}
$$

We note that direction dependent sensitivity of the image motion to changes in seeing quality is given by the constant coefficients $\left(K=\frac{\sigma^{2}}{\lambda^{2} r_{o}^{-5 / 3}}\right)$ :

$$
\begin{aligned}
& K_{\|}^{2}=2\left(0.179 D^{-1 / 3}-0.0968 d^{-1 / 3}\right) \\
& K_{\perp}^{2}=2\left(0.179 D^{-1 / 3}-0.145 d^{-1 / 3}\right)
\end{aligned}
$$

Finally the site quality defined as the long exposure FWHM (in radian) at the wavelength $\lambda$ and at zenith is related to the variance of the image motion measured in either direction $*$ at zenith angle $\gamma$ by:

$$
F W H M_{*}=0.976 \lambda^{-1 / 5}\left[\frac{\sigma_{*}^{2}}{K_{*}}\right]^{3 / 5}
$$


Actually, two seeing FWHM measurements are made (which improves the accuracy): a 'longitudinal' one in the direction $(\|)$ of the sub-aperture axis, and the 'transverse' one that is perpendicular $(\perp)$. The final result is the average of both at $\lambda=0.5 \mu m$ expressed in arcsec:

$$
F W H M=\frac{1}{2}\left(F W H M_{\|}+F W H M_{\perp}\right)
$$

\section{MISSION DESCRIPTION}

The ADIMM campaign began on Friday May 162003 (136), a team of the LPHEA carried out the first mission at Oukaïmeden site. The ADIMM instrument has been transported to Oukaïmeden. Unpacking and installation of the equipment did not present major problems. The experiment was operational on the night of Jun 14/15 (166). During one month, we are interested to correct all errors of the ADIMM software under LabWindows/CVI.

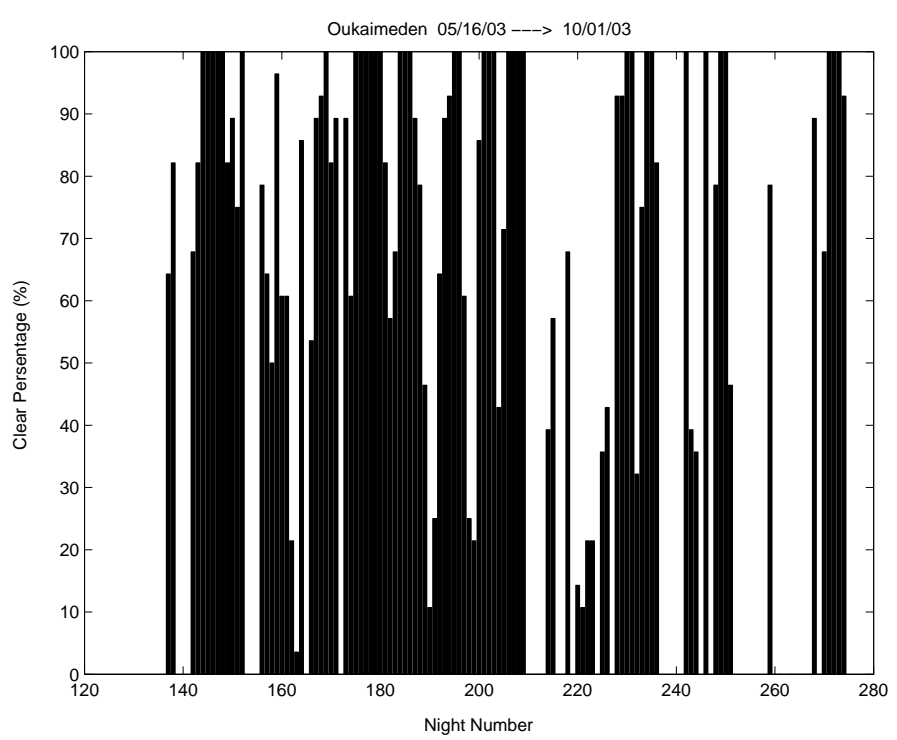

Figure 1. The summary of photometry for each night.

Since the beginning of this mission, the summaries of visual photometry are done. The photometry statistics for the campaign is given in the Fig. 1. We note that on 117 nights measured, 57 is of photometric quality. We consider the night as photometric if we have more than $80 \%$ of clear time.

In order to avoid the disturbance of the superficial terrestrial layer, it's necessary to put the instrument on a sufficient height. The currently international switchboards for the ADIMM is a height of $5 \mathrm{~m}$. We charged a local company for the construction of one $5 \mathrm{~m}$ tower (see plan Fig. ??).

We tryed to associate the instrumental constraints with the comfort of observations. So that we preferred to build some stairs to access to level of observation instead of a ladder traditionally constructed for this kind of platform. We also benefited of yard in order to construct a small cabin in a bottom of the tower in order to shelter the instrument and the observers (see photograph, Fig. 2)

All atmospheric parameters given in this report are calculated for a wavelength $\lambda=500 \mathrm{~nm}$ for observations near at zenith. Seeing corresponds to the full width at half maximum (FWHM) of a long-exposure stellar image in a long telescope. With these conditions, the 59 nights have produced a total 148.54 hours of monitoring of the turbulence parameters (Fig. 3). 


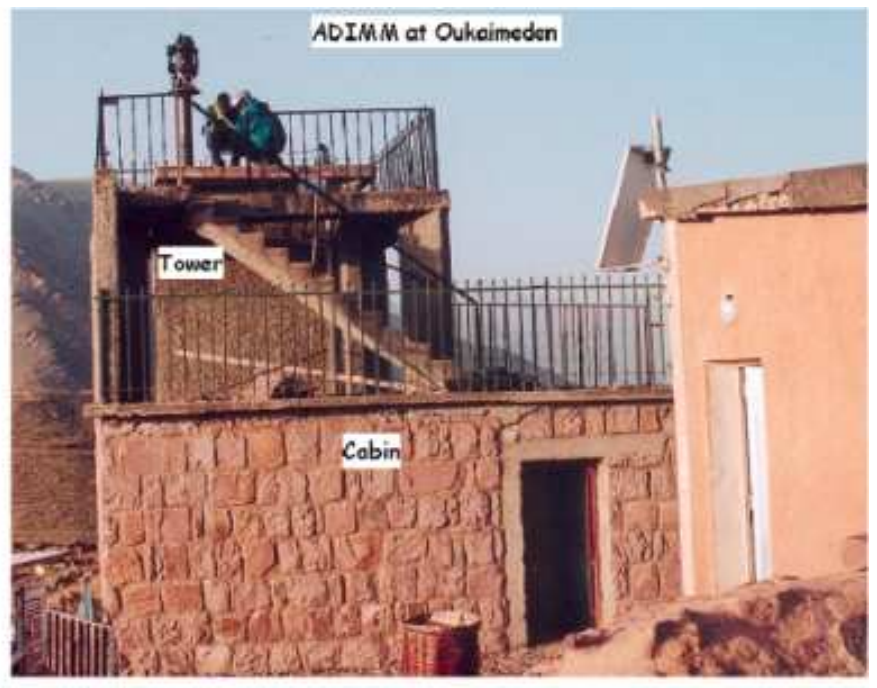

Figure 2. Tower and cabin'photograph.

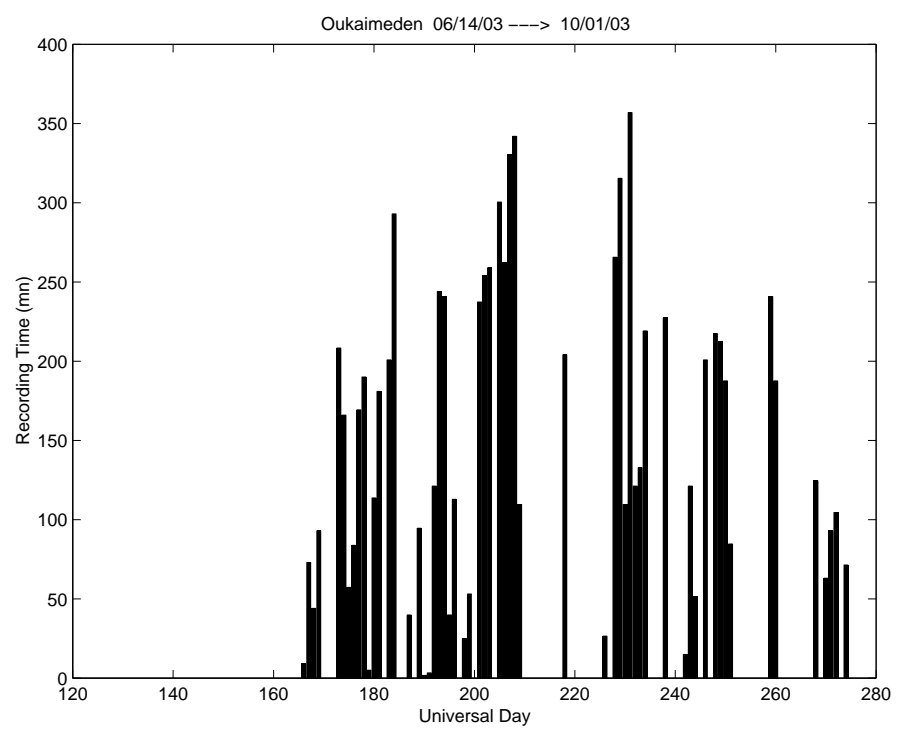

Figure 3. The distribution of the total measuring time over the 59 nights of the campaign.

\section{MARRAKESH ADIMM CHARACTERISTICS}

The technique employed is based on the statistical analysis of the differential tilt of the wavefront at two locations distant of about $14 \mathrm{~cm}$. The standard deviation of the differential slop of 100 short exposures with integration time $10 \mathrm{~ms}$ is converted into the FWHM of a long exposure stellar image by means of appropriate equations above. It requires however short exposures at a few ms intervals which can be obtained using either a fast read-out camera or non-destructive partial frame transfer.

The most relevant parameters which have been chosen in the design and construction of the our ADIMM are as follows (Fig.4 and Tables 1,2).

ADIMM was conceived as an instrument very similar to the ESO DIMM, with the same physical basis, but transformed to become a new generation of low cost with the components described above. A telescope and its electronics, loged on the tower at five meters above ground to escape from local ground turbulence (Fig. 6). 
Table 1. Telescope, masque and Prism characteristics

\begin{tabular}{|l|l|}
\hline Telescope Type & 8" Ultima 2000 Celestron \\
\hline Telescope Diameter & $203 \mathrm{~mm}$ \\
\hline Telescope Focal Length & $2032 \mathrm{~mm}$ \\
\hline Twin Pupil Diameter & $60 \mathrm{~mm}$ \\
\hline Distance Between Pupils & $140 \mathrm{~mm}$ \\
\hline Prism Deviation Angle & 40 arcsec \\
\hline
\end{tabular}

Table 2. Camera characteristics

\begin{tabular}{|l|l|}
\hline Model & ST-237A SBIG CCD \\
\hline Pixel Array & $657 \times 495$ pixels, $4.7 \times 3.6 \mathrm{~mm}$ \\
\hline Total Pixels & 307,000 \\
\hline Pixel Size & $7.4 \times 7.4$ microns \\
\hline Full Well Capacity & $20,000 e^{-}$ \\
\hline Dark Current & $5 e^{-} /$pixel $/ \mathrm{sec}$ at $0^{\circ} \mathrm{C}$ \\
\hline Shutter Exposure & 0.01 to 3600 seconds \\
\hline A/D Converter & $16 \mathrm{bits}$ \\
\hline A/D Gain & 0.72 \\
\hline Read Noise-Typical & $14 e^{-}$ \\
\hline Binning Modes & $1 \times 1,2 \times 2,3 \times 3$ \\
\hline Pixel Digitization Rate & $30 \mathrm{kHz}$ \\
\hline
\end{tabular}

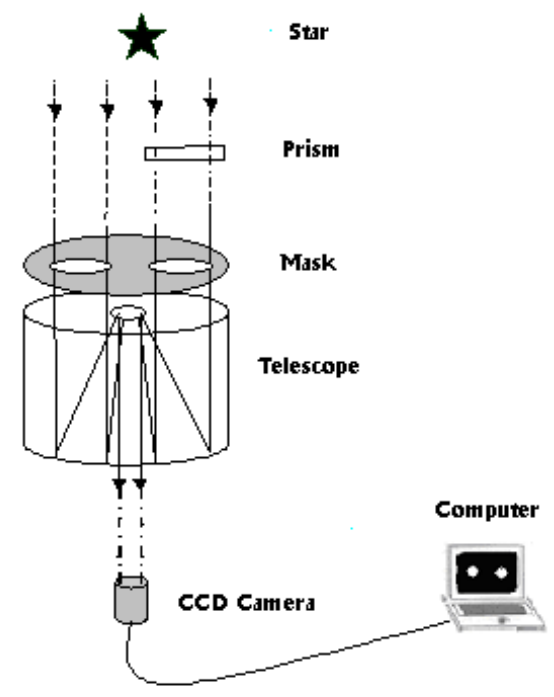

Figure 4. General configuration and components of the ADIMM. The PC is a Pentium III. The software iswritten in C running on a LabWindows under Windows NT/2000 


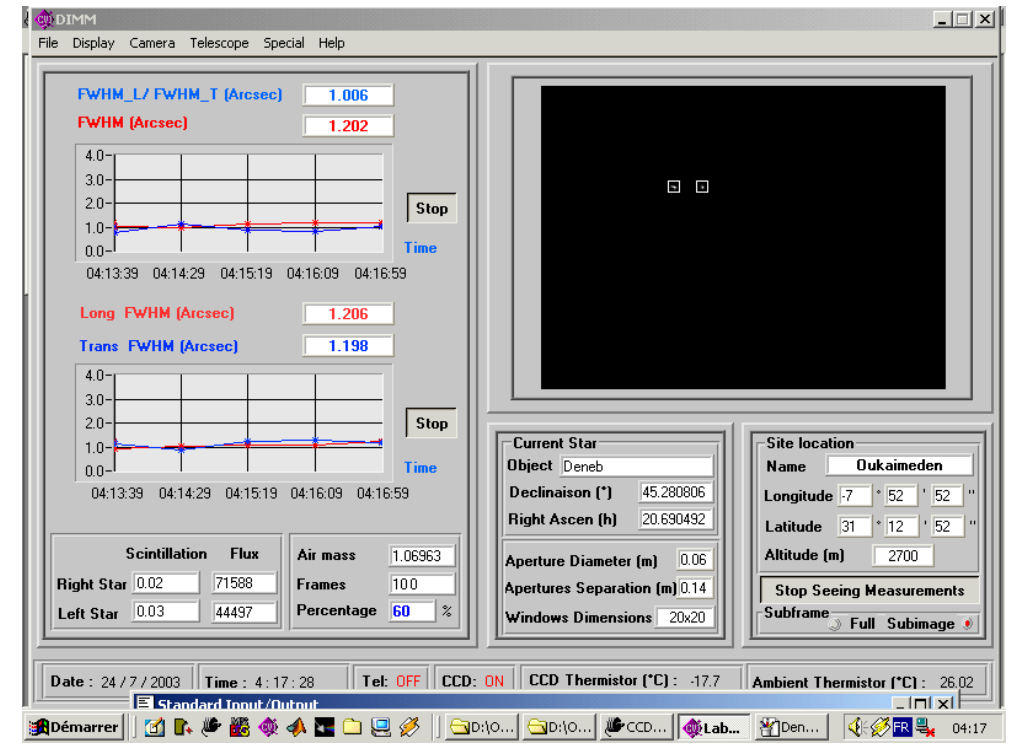

Figure 5. Main window of the Marrakesh ADIMM software.

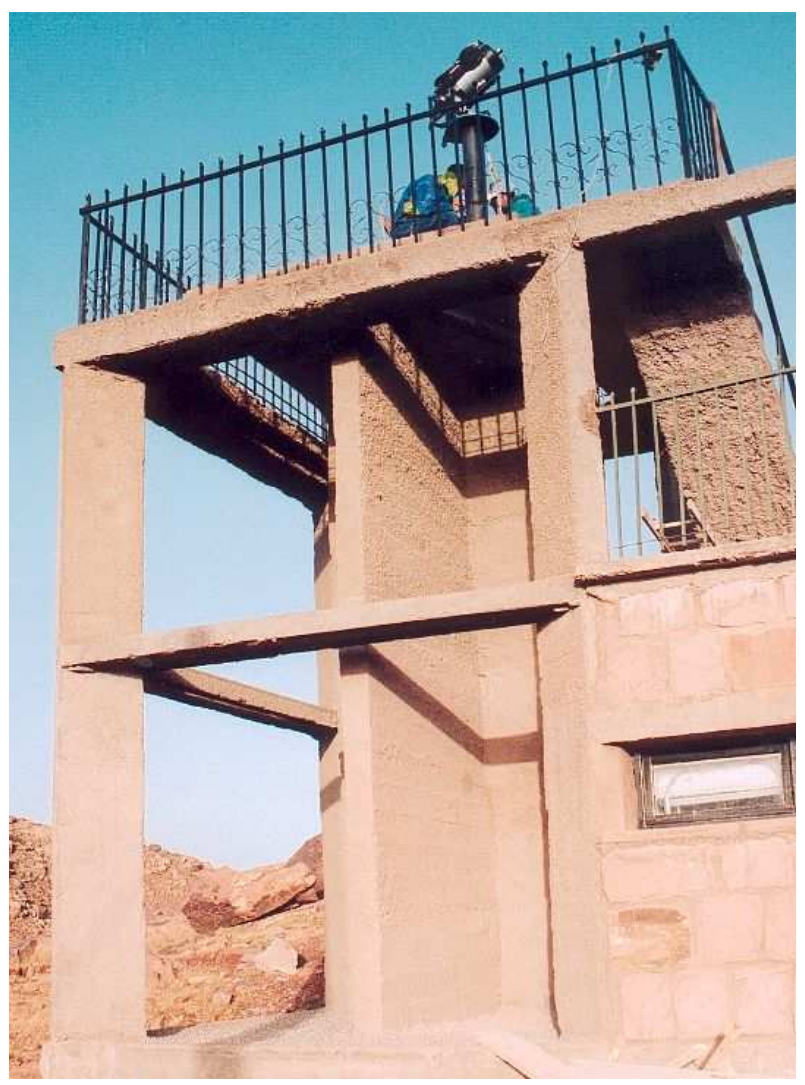

Figure 6. Photograph at the Oukaïmeden site showing ADIMM on "ESO" tower.

A CCD detectors and its associated electronics, attached to the telescope. A computer and software, used both for telescope control and data acquisition. The observers were housed in the ADIMM control room. 


\section{FIRST RESULTS OF MEASUREMENTS MADE AT OUKÄ̈MEDEN SITE}

The data available cover 59 nights between the $14^{\text {th }}$ of Jun and $1^{\text {th }}$ of October 2003 , they are presented in figures.1-2. Ideally, one data file is generated per star observed. In practice the working conditions are such that interruption, like power cuts, often force that a bad weather and star changing. Each line of the data file corresponds to a seeing measurement including: Universal time, longitudinal $\left(F W H M_{\|}\right)$, transverse $\left(F W H M_{\perp}\right)$, mean $\left(\left(F W H M_{\|}+F W H M_{\perp}\right) / 2\right)$ and ratio $\left(F W H M_{\|} / F W H M_{\perp}\right)$ seeing, flux, airmass. We only considered the stars close to the zenith (airmass $\leq 1.15$ ).

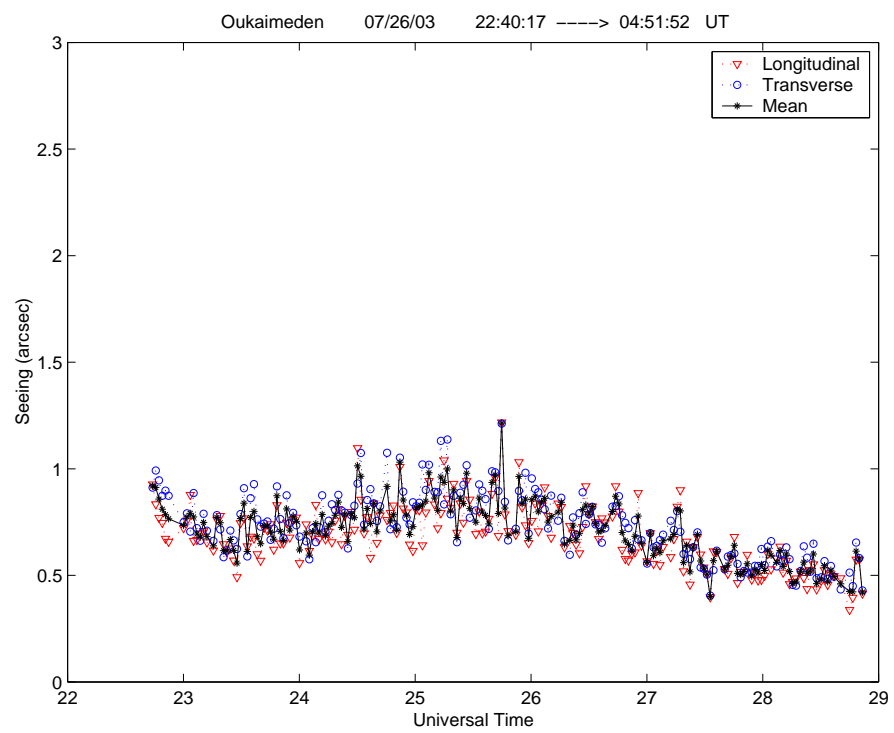

Figure 7. The temporal evolution of the Longitudinal, Transverse, and Mean seeing at Oukaïmeden site, during the whole night of 25 to $26 / 07 / 03$.

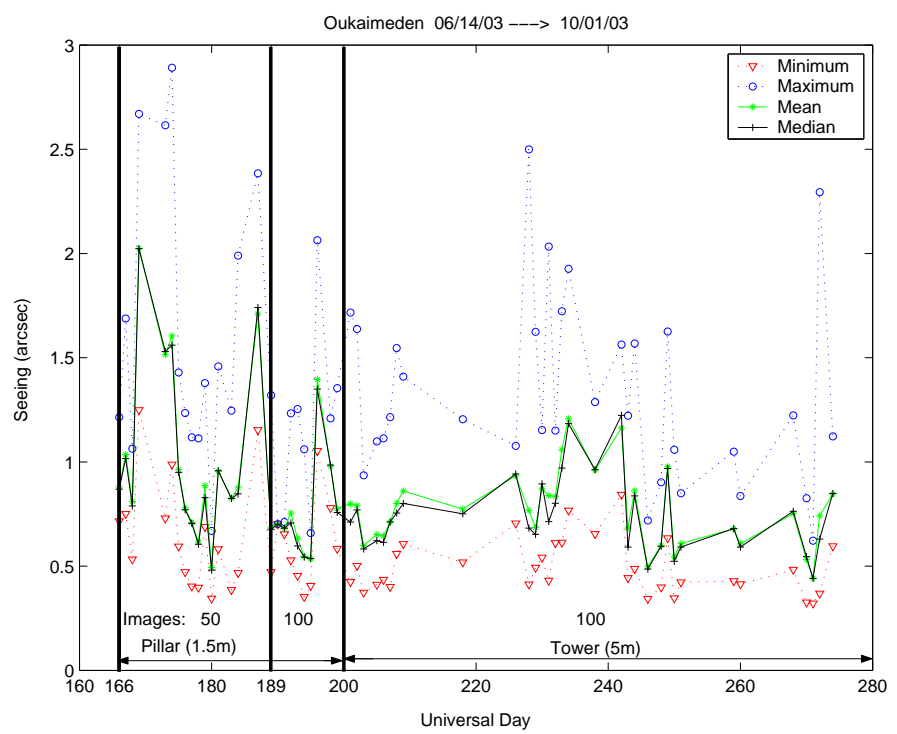

Figure 8. The ADIMM results obtained during Oukaïmeden mission from Jun 14 to October 012003 , evolution of the seeing values for each night.

The temporal evolution of the measurements provided at night $07 / 11 / 03$ is presented in Fig. 7 . All 
nights'seeing statistics are presented in Figs. 8,9 and 10. Measurement were taken with an Automated Differential Image Motion Monitor.

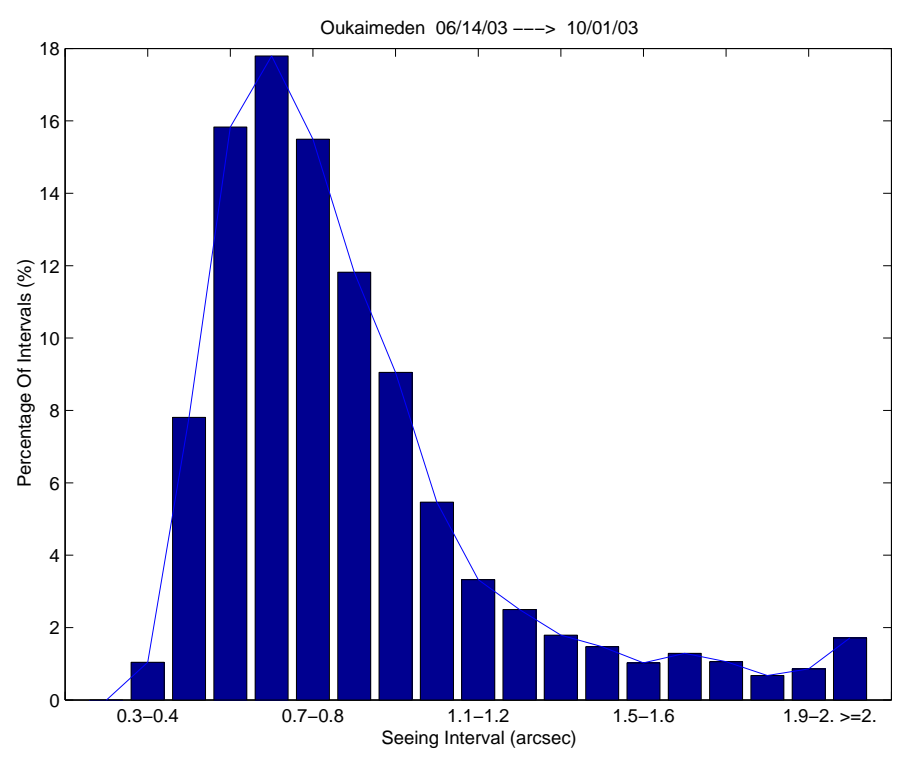

Figure 9. The distribution of intervals of seeing from the database described here.

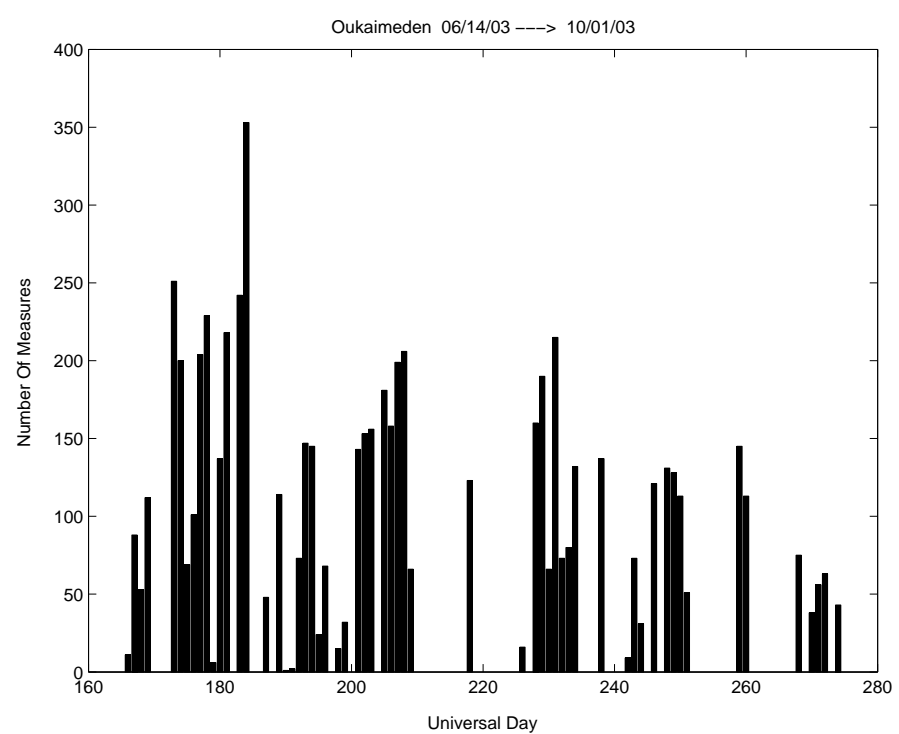

Figure 10. The distribution of the total measuring data during 59 nights at the Oukaïmeden site.

In Fig. 8 the seeing values are high between 06/15/03 (166) and 07/18/03 (200), because the ADIMM was installed on top of pillar with the height of telescope $1.7 \mathrm{~m}$ above ground, and a seeing estimate is produced from data accumulated during a total of 50 samples of 06/15/03 (166) at 07/08/03 (189) and 100 images of 07/09/03 (190) at $07 / 18 / 03$ (200). But, of the $07 / 20 / 03$ (201), the ADIMM is installed on a $5 \mathrm{~m}$ high tower, in order to avoid the near-ground turbulence as much as possible.

From the whole campaign the mean seeing is 0.84 ", the median value is 0.75 " and $9 \%$ of the all data the seeing is better than 0.5 ". The best seeing measured is 0.32 ". 


\section{CONCLUSION}

In this preliminary survey, we are presenting, for the first time, our automted DIMM (ADIMM). We are also presenting the firsts results of seeing measured on a rather long period. These first four month of measurement showed that the instrument is extremely reliable. We didn't record any breakdown of working indeed. The data were taken on a $5 \mathrm{~m}$ tower to avoid turbulence produced by the surface layer. Median and mean seeing values respectively of 0.75 and 0.84 arcsec were achieved over the campaign. This values of the seeing require a finer analysis, however it allow us to situate the site among some astronomical observatories known for their good seeing such those of Chil or Canary islands.

We are working now on a publication that will present the results of one year of seeing measurment by ADIMM on the Oukaïmeden site with deeper analysis. The instrument we built, could be duplicate and use for the futur site prospection in Morroco or every place preselected for the the futur Extremly Large Telescope site prospection.

\section{ACKNOWLEDGMENTS}

We are very indebted to Mark Sarazin and Jean Vernin for helped us by there important remarks and advises. We thank very much the agencies who financed the instrument and site testing campagne (contracts: ESO Num.69651/ODG/02/9005/GWI, Morrocan PARS Num.66/SDU). This study was made possible, also thanks to Ouchaou Omar, our local technician, converted in observant astronomer, for this opportunity. He play a major role for the succes of this campaign, we are very thankful to him.

\section{REFERENCES}

1. Benkhaldoun, Z., Kadiri, S., Lazrek, M., \& Touma, H., Solar Physics, 133, 61, (1991).

2. Benkhaldoun, Z., Kadiri, S., Lazrek, M., \& Vernin, J., Experimental Astronomy, 2, 345-356, (1993).

3. Benkhaldoun, Z., Ph.D thesis, Cadi Ayyad University, Marrakech, Morocco, (1994).

4. Benkhaldoun, Z., \& Siher, E. A., SOHO6/GONG98 Workshop, Boston (USA), ESA SP-418,109-113, (1998).

5. Benkhaldoun, Z., ASP Conference Series, 414-423, 266, (2002).

6. Hill, F., Solar Physics, 152, 321, (1994a).

7. Hill, F., Solar Physics, 152, 351, (1994b).

8. Jabiri, A., Benkhaldoun, Z., Vernin, J., \& Muoz-Tuon, C., A \& A, 271, 284, (2000).

9. Kadiri, S., Ph.D thesis, Nice University, (1983).

10. Sarazin, M., \& Roddier, F., A \& A, 227, 294, (1990).

11. Siher, E.A., \& Benkhaldoun, Z. , In revision in New Astronomy, (2003).

12. Siher, E.A., Benkhaldoun, Z., \& Fossat, E., To appear in Experimental Astronomy, (2003).

13. Ziad, A., Jabiri, A., Benkhaldoun, Z., Martin, F., Conan, R., Lazrek, M., \& Borgnino, J., A \& A, 365, 324, (2001). 